\title{
Structural and entropic insights into the nature of the random-close-packing limit
}

\author{
A. V. Anikeenko and N. N. Medvedev \\ Institute of Chemical Kinetics and Combustion, Siberian Branch of the Russian Academy of Sciences, 630090 Novosibirsk, Russia \\ T. Aste \\ Department of Applied Mathematics, The Australian National University, 0200 Canberra, ACT, Australia \\ (Received 14 September 2007; revised manuscript received 27 November 2007; published 3 March 2008)
}

Disordered packings of equal sized spheres cannot be generated above the limiting density (fraction of volume occupied by the spheres) of $\rho \simeq 0.64$ without introducing some partial crystallization. The nature of this "random-close-packing" limit (RCP) is investigated by using both geometrical and statistical mechanics tools applied to a large set of experiments and numerical simulations of equal-sized sphere packings. The study of the Delaunay simplexes decomposition reveals that the fraction of "quasiperfect tetrahedra" grows with the density up to a saturation fraction of $\sim 30 \%$ reached at the RCP limit. At this limit the fraction of aggregate "polytetrahedral" structures (made of quasiperfect tetrahedra which share a common triangular face) reaches it maximal extension involving all the spheres. Above the RCP limit the polytetrahedral structure gets rapidly disassembled. The entropy of the disordered packings, calculated from the study of the local volume fluctuations, decreases uniformly and vanishes at the (extrapolated) limit $\rho_{K} \simeq 0.66$. Before such limit, and precisely in the range of densities between 0.646 and 0.66 , a phase separated mixture of disordered and crystalline phases is observed.

DOI: 10.1103/PhysRevE.77.031101

\section{INTRODUCTION}

Sphere packings have been studied for centuries to model natural structures both at the atomic level and at the macroscopic level [1]. One of the main quests in these studies is to understand the nature of the transition between disorderedand ordered-crystalline packings. It is known that the densest packing of equal spheres has a fraction of volume occupied by the balls with respect to the total volume (packing fraction or density, $\rho$ ) equal to $\rho=\frac{\pi}{\sqrt{18}} \approx 0.74$ [1]. Such maximal density can be realized in infinitely many ways, with two common examples being the face-centered-cubic lattice (fcc) and the hexagonal-closed-packed structure (hcp). All these maximally dense packings are based on stacking closepacked two-dimensional hexagonal layers of spheres. The class of such maximally dense layered packings are known as Barlow packings (named after the mid-19th century scientist who explored several possible stackings of spheres in an attempt to explain the atomic origin of crystal shapes [1]). They are the most common crystalline structures in atomic systems like heavy metals and solid noble gases, and they are also commonly observed in colloids. Atomic systems can also have noncrystalline phases, but these are in general metastable states and the system will eventually relax into the crystalline phase, which is more stable thermodynamically. Conversely, particles of nonmicroscopic sizes (athermal systems with typical sizes above $50 \mu \mathrm{m}$, often called under the general name of "granular materials") reveal a strong tendency to avoid crystallization despite the fact that this is the most compact arrangement and therefore the one with the lowest potential energy (gravitational). To describe the nature of such noncrystalline packings and to understand the mechanisms that prevent and induce crystallization is one of the major challenges in present day research on packings and granular materials. Empirical studies [1-6] show that mechanically stable packings of equal spheres can be pro- duced at different densities in the rather broad range between the two limiting densities $\sim 0.555$ [called random loose packing $(\mathrm{RLP})$ ] and $\sim 0.646$ [called random close packing $(\mathrm{RCP})]$. The fact that noncrystalline packings of equal spheres cannot be packed tighter than the limiting density of $\sim 0.64$ was first observed in the middle of the last century by Bernal in experiments with steel balls [7]. However, the microscopic origin of this bounding density is still unexplained and even the existence of such a limit is still a controversial issue [8].

Some important improvements in our understanding of the structural changes occurring at the RCP limit were recently reported in a paper by two of the authors [9]. The paper considered the structure of numerically generated sphere packings in terms of Delaunay simplexes. These simplexes are unambiguously and uniquely defined for any (regular or disordered) set of points in space. They define configurations of quadruples of "atoms," and they are the simplest three-dimensional elements to which a threedimensional packing can be reduced. Delaunay simplexes represent a mosaic covered space of a sample, so if we select simplexes with a given shape, the clusters of such simplexes give a design on the mosaic to reveal a structural motif $[10,11]$. It was observed in Ref. [9] that the volume fraction occupied by Delaunay simplexes with "quasiregular" shapes increases with increasing density up to Bernal's RCP limit. At this stage a structural transition was observed, with the volume fraction occupied by clusters of tetrahedra (polytetrahedra) passing through a sharp maximum while the fraction of spheres was involved in tetrahedra saturates. The position of this maximum was estimated at 0.646. That study clearly indicated that some structural reorganization is occurring at the RCP limit.

In this work, we consider in further detail the nature of this transition by comparing different measures to identify the quasiregular tetrahedra and by extending the analysis to experimental systems. We confirm the polytetrahedral struc- 
ture of disordered packings, we retrieve the drastic behavior of the clusters of tetrahedra at the limiting density, and we demonstrate that the structure of packings in physical experiments is very similar to the structure observed in numerically simulated ideal hard spheres packings. These are important results that strongly support the original finding [9] that a structural transition is taking place at the RCP limit. However, questions are is still left open: why these structural changes happen and what is the nature of such a transition? In the second part of this paper we use a statistical mechanics approach to calculate the system's entropy and we show that immediately above the RCP density a rapid increase in the entropy is observed. We identify such change in entropy with the formation of mixed disordered-crystalline configurations which are coexisting in the range of densities between 0.646 and 0.66 . The extrapolation of the disordered packings' entropy identifies the limiting density $\rho_{K} \simeq 0.66$ at which such entropy will vanish. We discuss these observations by drawing similarities with glass-forming supercooled liquids.

The structure of the paper is the following: in Sec. II we present in detail the analyzed systems and their preparation methods; the Delaunay and Voronoï decompositions are introduced in Sec. III; methods to classify Delunay shapes are discussed in Sec. IV; the evidence of a structural transition occurring at the RCP limit is presented in Sec. V; in Sec. VI a way to calculate the system's entropy is introduced; the evidence of a transition taking place above the RCP limit is reported in Sec. VII and its nature is discussed; finally, conclusions are given in Sec. VIII.

\section{METHODS AND MATERIALS}

\section{A. Computer simulations of sphere packings}

We study large sets of numerically simulated hard sphere packings with packing densities ranging from 0.53 to 0.71 . Each packing contains 10000 identical spheres in a cubic box with periodic boundary conditions. In order to test if the packing structures are independent of the preparation algorithm we produced sphere packings using two different numerical approaches. The majority of the packings (more than 200) were obtained using a modified Jodrey-Tory algorithm that employs "repulsion" of overlapping spheres with gradual reduction of their radii [12-14]. In this algorithm the initial configuration is a set of identical overlapping spheres uniformly distributed in the box. The algorithm attempts to reduce overlaps between spheres by shifting overlapping spheres and gradually shrinking their radii. This procedure is carried on until all overlaps are removed. This algorithm can easily produce disordered packings with densities up to the limiting RCP value of $\sim 0.646$, but it can also produce more dense systems containing crystalline inclusions up to densities around 0.66 . Higher densities can be reached by using the result of an earlier run (with enlarged diameters) as the starting configuration.

Another set of packings (about 70) was produced in the range of densities from 0.54 to 0.67 by using a modified Lubachevsky-Stillinger algorithm [15]. In this algorithm the initial configuration is also random but overlaps are forbidden. The simulation consists of an event-driven Newtonian dynamics in which the spheres are considered perfectly elastic. During the simulation, the radii of the spheres are gradually increased until a "jammed configuration" where the radii cannot be enlarged anymore is reached. In these simulations the principal control parameter is the growth rate for sphere radii. Small values of growth rates will result in crystallization. To avoid crystallization the growth rate should be rather large, forcing the packing into "jammed" noncrystalline structures $[8,16]$. For this study, we have generated packings of $N=10000$ spheres jammed up to a maximal reduced pressure of $10^{12}$ using a growth rate for sphere radii in the range between $2 \times 10^{-5}$ and 0.2 [17].

\section{B. Sphere packings experiments}

The numerical results were compared with a set of six experiments from the AAS database on disordered packings [18] which contains structural data from experimental sphere packings obtained by $\mathrm{x}$-ray computed tomography. In particular, we analyze six samples (A-F) composed of acrylic beads in air prepared within a cylindrical container with an inner diameter of $55 \mathrm{~mm}$ and filled to a height of $\sim 75 \mathrm{~mm}$ $[6,19,20]$. Samples A and C contain $\sim 150000$ beads with diameters $d=1.00 \mathrm{~mm}$ and polydispersity within $0.05 \mathrm{~mm}$. Whereas samples B, D-F contain $\sim 35000$ beads with diameters $d=1.59 \mathrm{~mm}$ and polydispersity within $0.05 \mathrm{~mm}$. The geometrical investigation of the packing structure was performed over a central region at 4 sphere-diameters away from the sample boundaries. The density (packing fraction) of each of the samples is A, $\rho \sim 0.586 ; \mathrm{B}, \rho \sim 0.596 ; \mathrm{C}, \rho$ $\sim 0.619$; D, $\rho \sim 0.626$; E, $\rho \sim 0.630$; and F, $\rho \sim 0.640$. The two packings at lower densities (A, B) were obtained by placing a stick in the middle of the container before pouring the beads into it and then slowly removing the stick [1]. Sample C was prepared by gently and slowly pouring the spheres into the container. Whereas sample D was obtained by a faster pouring. In sample $\mathrm{E}$ a higher density was achieved by gently tapping the container walls. The densest sample (F) was obtained by a combined action of gentle tapping and compression from above (with the upper surface left unconfined at the end of the preparation). To reduce boundary effects, the inside of the cylinder was roughened by randomly gluing spheres to the internal surface. In this paper we also present data concerning 12 samples of glass beads with diameters of $0.25 \mathrm{~mm}$ prepared in water by means of the fluidized bed technique [18,21,22]. Packing densities between 0.56 and 0.60 were obtained by using different flow rates. After each flow pulse, the particles sedimented forming a mechanically stable packing. Packings created in this way are in a stationary state with packing densities which are independent of the preparation history and fluctuate around average values with smaller densities for higher flow rates. This kind of technique is probably the best suited for applying statistical mechanics approaches $[21,22]$.

\section{DELAUNAY AND VORONOÏ DECOMPOSITIONS}

Our first aim is to characterize the structure of disordered sphere packings and quantify structural properties and struc- 
tural changes associated with different densities. The first step in the quest for a structural characterization is to identify local configurations and quantify their similarities and relative occurrences. To this end a convenient approach is to decompose the system into a set of local "units" whose properties can then be analyzed statistically. There exists a general approach, used in geometry, which allows us to unambiguously decompose any arbitrary system of discrete points into a space-filling set of local "units" or cells. Such an approach is the Voronoi-Delaunay tessellation (decomposition), well-known both in physics [23] and mathematics [24]. This method exploits an evident geometrical fact that for each point in a set of points embedded in a given metric space it is always possible to distinguish the portion of space closest to such a point with respect to any other point in the set. This region is called the Voronoï polyhedron (cell, region) and the space-partition built from the assembly of all Voronoï cells is called the Voronoï tessellation or Voronoï diagram [24]. For any Voronoï tessellation, there exists a dual tessellation called the Delaunay tessellation, which consists of Delaunay simplexes (irregular tetrahedra, in the general case) whose vertices are the quadruples of closest points in the set. The names of these constructions derive from the mathematicians that posed the mathematical foundations of the methods: Voronoï (1868-1908) (who explored in detail the properties of these tessellations by using analytical methods for lattice systems) and Delaunay (1890-1980) (who proved the correctness of Voronoï's main theorems for points positioned at random in space) [25-28]. The Voronoï cell delimits the "region of influence" around each point. For packings of identical spheres, the volumes of the Voronoï cells constructed from the set of sphere centers are associated with the local packing density.

\section{SHAPE CHARACTERIZATION}

In order to characterize the packing structure we can use the simplicial decomposition defined by the VoronoïDelaunay construction. For a quantitative characterization, we first need to build a simple instrument to measure the shape of each simplex. In particular we might look at the "distance" between a given simplex and a perfect (regular) tetrahedron. Several approaches have been suggested to characterize the proximity of a simplex to a perfect tetrahedron [10,29-32]. In this paper we will discuss three different methods that embrace a significant range of possibilities.

\section{A. Edge differences, $T$ measure}

Let us start with a rather old and simple method in which the irregularity of the tetrahedron is quantified by summing over the average square of the simplex edge-length differences [10],

$$
T=\frac{1}{15 \vec{l}^{2}} \sum_{i<j}\left(l_{i}-l_{j}\right)^{2}
$$

where $l_{i}, l_{j}$ are the lengths of the simplex edges, and $\bar{l}$ is the mean edge length. In a perfect tetrahedron all edges have

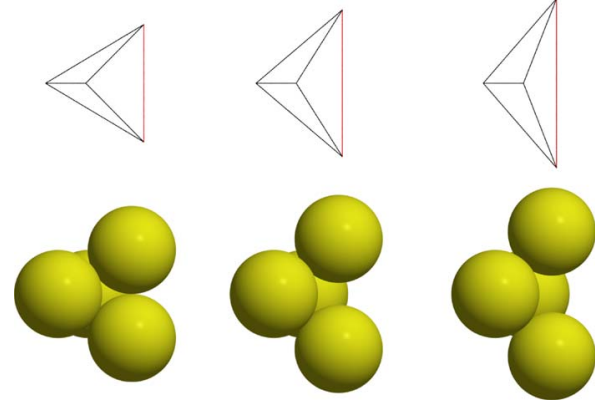

FIG. 1. (Color online) Typical simplexes in dense packings of equal spheres: (a) perfect tetrahedron (it has $T=0, d_{t}=0, \delta=0$ ); (b) an example of "a boundary" simplex with quasiregular tetrahedral shape (with $T \sim 0.018, d_{t} \sim d_{q}, \delta \sim 0.25$ ); and (c) perfect quartoctahedron (a quarter of an octahedron, with one edge $\sqrt{2}$ longer than the others, $T=0.05, d_{t}=0.179, \delta=0.41$ and $\left.d_{q}=0\right)$, see text.

equal length and $T$ is equal to zero. More generally, small values of $T$ correspond to simplexes which are close to a perfect tetrahedron. Conversely, large values of $T$ indicate significant deviations from regularity.

We now want to identify a bound on the value of $T$ which defines a class of tetrahedra that are regular enough and therefore can be considered "quasiperfect tetrahedra." In Refs. [11,33] this measure was calibrated using models of a fcc crystal at different temperatures (at different degrees of perturbation). It is known that this crystal structure (as any Barlow packing) can be reduced to a tiling with elementary tiles made of a mixture with two perfect tetrahedra and one octahedron. At finite temperatures they are distorted, but as long as the crystal regularity is retained the two main classes of Delaunay simplexes, tetrahedra and quartoctahedra (quarters of octahedra), are present (see Fig. 1). It was shown in Refs. [11,33] that the boundary value $T_{b}=0.018$ divides the two classes of simplexes in the calibrating models. This is therefore the threshold value for the $T$ measure which we will use in the present paper.

\section{B. Procrustean distance, $d$ measure}

From the perspective of mathematical shape theory $[34,35]$, the proximity of an arbitrary simplex to a given reference shape is estimated by the degree of coincidence upon their superposition. To this end one can compute the total mean square deviation $d^{2}$ between the corresponding vertices of the optimally superimposed simplexes. The quantity $d$ is called the Procrustean distance between the two simplexes. Let $\left\{\mathbf{x}_{1}, \mathbf{x}_{2}, \mathbf{x}_{3}, \mathbf{x}_{4}\right\}$ and $\left\{\mathbf{y}_{1}, \mathbf{y}_{2}, \mathbf{y}_{3}, \mathbf{y}_{4}\right\}$ be, respectively, the coordinates of the vertices of two simplexes, the square of Procrustes distance between them is

$$
d^{2}=\min _{\mathbf{R}, \mathbf{t}, P}\left\{\frac{1}{4} \sum_{i=1}^{4}\left\|\mathbf{y}_{\mathbf{i}}-\left(\mathbf{R} \mathbf{x}_{\mathbf{i}}+\mathbf{t}\right)\right\|^{2}\right\},
$$

where the minimum is calculated over all three-dimensional rotations $\mathbf{R}$, translations $\mathbf{t}$, and all possible combinatorial mappings between vertices of the simplexes $(P)$. For a given correspondence (mapping) between vertices, it is possible to 
calculate analytically the Procrustean distance and there are several algorithms to solve such a least-squares problem (see Ref. [36] for details). The measure $d$ allows us to compare a simplex to any reference shape. For instance, it is possible to calculate the distance from a given simplex to both the perfect tetrahedron $d_{t}$ and to a quartoctahedron $d_{q}$ (a quarter of an octahedron with one edge $\sqrt{2}$ longer than the others, see Fig. 1). This also suggests that a natural choice to classify quasiperfect tetrahedra is to select simplexes with Procrustean distances to a perfect tetrahedron smaller than their distance to a perfect quartoctahedron, i.e.,

$$
d_{t}<d_{q} .
$$

The distance between perfect tetrahedron and quartoctahedron is equal to 0.17936 [31].

\section{Maximal edge length, $\delta$ measure}

A very simple but effective way to determine how close an irregular simplex is to a perfect tetrahedron consists of calculating the length of the longest edge $e_{\max }$. This method of selecting tetrahedral simplexes was used by Hales in his proof of the Kepler conjecture [32]. This approach seems especially suitable in packings of hard spheres with equal (unit) diameters, where the minimal possible length of the simplex edge is equal to 1 . In this case, a value of $e_{\max }$ close to unity unequivocally indicates that all edges are close to 1 and therefore the simplex is close to a regular tetrahedron. A convenient measure of the simplex shape is therefore the difference between the lengths of the maximal and the minimal edges: $\delta=e_{\max }-1$ [9]. Small values of $\delta$ unambiguously indicate that the shape of the simplex is close to a perfect tetrahedron, while large values of $\delta$ correspond to substantially distorted shapes. In the proof of the Kepler conjecture, Hales chose the maximal edge length 1.255 as the upper bound for quasiperfect tetrahedra which in our notation corresponds to $\delta=0.255$. In this paper, we will also use this threshold value which is in between the perfect tetrahedron $(\delta=0)$ and the quartoctahedron $(\delta=0.414 \ldots)$, consistent with the previous measures.

It is important to remark that the $\delta$ measure is strictly related to the two previous measures. One can verify that for dense packings of hard spheres all these measures pick practically the same tetrahedral simplexes. For instance, in disordered packing at density 0.64 we estimated that the conditions $T<0.018$ and $\delta<0.255$ select the same simplexes with an overlap of $90 \%$, and the coincidence rate increases with the onset of crystallization. Thus each of the measures reliably picks tetrahedra with shapes close to perfection. Some ambiguity is observed only for simplexes with boundary shapes, but they are not critical in our analysis.

\section{STRUCTURAL REORGANIZATIONS AT THE RANDOM CLOSE PACKING LIMIT}

\section{A. Fraction of quasiperfect tetrahedra}

For each packing we calculate all the Delaunay simplexes and select the quasiperfect tetrahedral shapes accordingly with the criteria discussed in the previous section. Figure 2

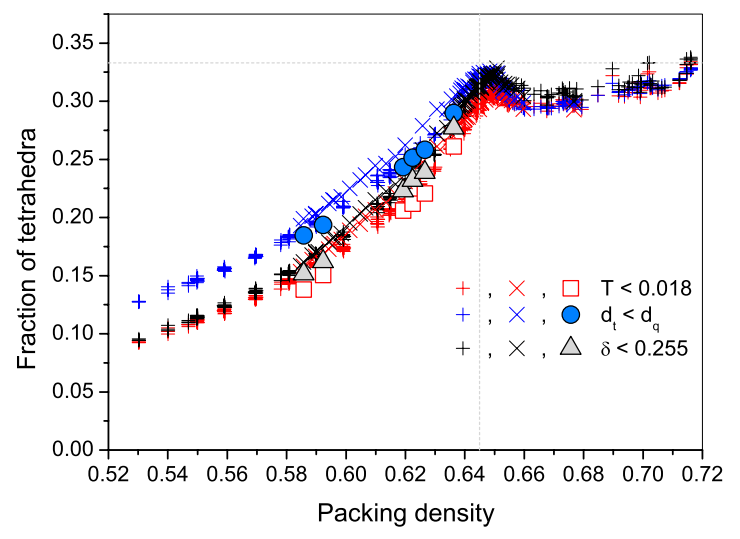

FIG. 2. (Color online) Fraction of Delaunay simplexes with quasiperfect tetrahedral shape as a function of packing density. From the top to bottom: $d_{t}<d_{q}$ (blue, online only), $\delta<0.255$ (black), $T$ $<0.018$ (red, online only). The symbols correspond, respectively, to dry acrylic beads experiments $(\square, \bigcirc$, and $\triangle$, respectively, for $T$ $<0.018, d_{t}<d_{q}$, and $\delta<0.255$ ); numerical simulations by JodreyTory algorithm $(+)$; and numerical simulations by LubachevskyStillinger algorithm $(\times)$. The vertical line marks the density $\rho$ $=0.646$ which has been individuated as the best estimate for the position of the maximum. The horizontal line marks the value of $1 / 3$ that corresponds to the fraction of tetrahedra in the densest crystals.

shows the behavior of the fraction of quasiperfect tetrahedra as a function of the packing density for all numerical models and experiments A-F. One can verify that the general trends are comparable for all three measures of shape described above. Only the Procrustean distance $\left(d_{t}<d_{q}\right)$ tends to overestimate the fraction of tetrahedra at low densities. Indeed, this criterion can pick rather distorted simplexes that are far away from a perfect tetrahedron, but are even farther from a perfect quartoctahedron. Note that each point on a curve corresponds to an independent packing. The good coincidence of points at similar densities illustrates the representativeness of our computer models. The agreement between experimental and numerical results is also evident.

The fraction of tetrahedra rapidly grows with increasing density, reaching a value around $30 \%$ when approaching the critical density of $\rho \sim 0.646$. Interestingly, further increase of the density has little effect on the fraction of tetrahedra. Note that the fraction of quasiperfect tetrahedra at $\rho \sim 0.646$ is close to $1 / 3$, which corresponds to the fraction of perfect tetrahedra in Barlow packings. Such a coincidence of the fraction of quasiperfect tetrahedra with the ones in the densest crystalline structure deserves special attention as this can shed light on the physical meaning of the class of quasiregular tetrahedra. But the problem is not straightforward. The question is, which is the maximum fraction of quasiperfect tetrahedra which can be present in a dense packing of equal spheres? It seems reasonable to conjecture that the fraction of $1 / 3$ is an upper bound. However, a recent work [37] seems to suggest that some classes of tetrahedral packings might reach larger fractions. Moreover, the body-centeredcubic (bcc) crystal consists of Delaunay simplexes which are all quasiregular tetrahedra according to two of our criteria $\left(T_{b c c}=0.011, \delta_{b c c}=0.15\right)$. It must be also noted that the frac- 


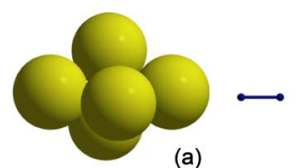

(a)

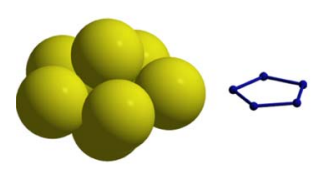

(c)

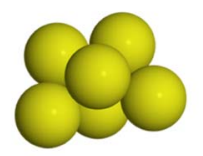

(b)

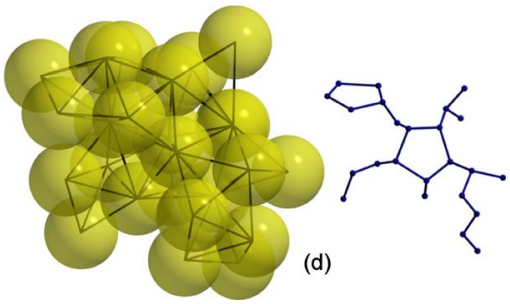

FIG. 3. (Color online) Examples of polytetrahedral aggregates (clusters of face-adjacent tetrahedra). (a) Two tetrahedra; (b) three tetrahedra; (c) a ring of five tetrahedra; and (d) a typical cluster in a dense disordered packing. The graphs on the right sides show the motives of the tetrahedra clusters: the points mark the centers of tetrahedra and the lines indicate that they are adjacent through a common face. Clusters of three and more face-adjacent tetrahedra are impossible in Barlow packings and lattices in general (incompatible with translational symmetry).

tion of tetrahedra depends also on the softness of the spheres. For instance, we have found that in Lennard-Jones glasses one can have up to $40 \%$ of such quasiregular tetrahedra.

\section{B. Fraction of polytetrahedral aggregates}

We have established so far that in disordered packings of equal sized spheres the fraction of quasiperfect tetrahedra increases during densification and reaches a plateau around $30 \%$ when the random close packing limiting density is overcome. We now want to understand how these quasiperfect tetrahedra can aggregate in more complex structures. To this end we analyze clusters of face-adjacent tetrahedra (Fig. 3). In particular, we consider clusters built from three or more face-adjacent quasiperfect tetrahedra and we call such structures polytetrahedra $[9,38]$. Isolated tetrahedra and pairs of tetrahedra [bipyramids, Fig. 3(a)] are omitted as they are found in fcc and hcp structures (see Fig. 4). We can associate a graph to such polytetrahedra aggregates. In such a graph a

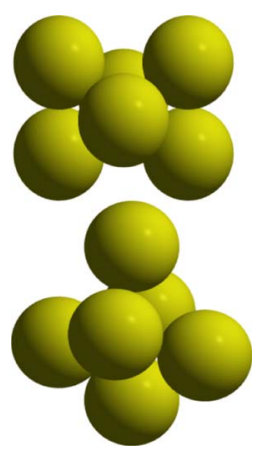

FIG. 4. (Color online) (Top) Two edge-adjacent tetrahedra (present in the fcc lattices); they have edge-adjacent quartoctahedra between them. (Bottom) Two face-adjacent tetrahedra (hcp structures); they have neighbor face-adjacent quartoctahedra.
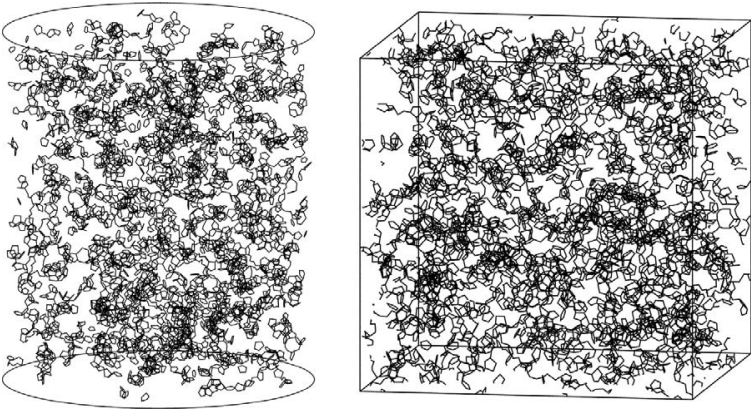

FIG. 5. Illustration of spatial distribution of the polytetrahedral clusters in packings of hard spheres at $\sim 0.64$ in experiments (left) and computer models (right). To simplify pictures only skeletons of the polytetrahedra are shown. Tetrahedral simplexes are selected according the measure $T<0.018$.

vertex represents the center of a quasiperfect tetrahedron and a segment between two vertices is inserted when two quasiperfect tetrahedra are sharing a face [Fig. 3 (right sides)]. In general, such graphs have the form of branching chains and five-edges cycles which combine in various "animals" [11,38], see Figs. 3(d) and 5. In mathematical jargon, such a representation of clusters of the selected Delaunay simplexes is called "site-coloring on the Voronoï network." Indeed, because of the duality of the Delaunay and Voronoï tessellations, the center of any Delaunay simplex is a vertex of the Voronoï network, and a common edge is connecting two neighboring vertexes in the Voronoï network [10,23].

Figure 5 shows the graphs of polytetrahedral clusters inside a numerically generated sample and an experimental sample both at densities $\sim 0.64$. We see many five-member rings revealing the "5-symmetry nature" of the disordered packings discussed by Bernal in his work [7]. Visual analyses of these clusters reveal that they are rather irregular. Note that there are no clusters structured as dodecahedra (polyhedron with 12 five-member faces) which would correspond to icosahedral local configurations of spheres. This fact is an additional argument that "icosahedral local order" is not typical in disordered packings of identical spheres [20,39-41]. Note that we do not observe practically any six-member rings, although our class of tetrahedra allows distortions of shape to organize such rings (e.g., a part of the Delaunay simplexes in the bcc structure are arranged in such rings). In disordered packings, six-member rings of tetrahedra seem not to be preferable.

In Fig. 6 the fraction of tetrahedra which are part of polytetrahedra aggregates is reported as a function of the packing density. We observe that the polytetrahedra aggregates rapidly grow with increasing density. Upon approaching the Bernal's limit density, the fraction of polytetrahedral aggregates also accounts for about $30 \%$ of all Delaunay simplexes indicating that almost all quasiperfect tetrahedra are involved in such polytetrahedral aggregates. However, after the RCP density the fraction of the tetrahedra belonging to polytetrahedral aggregates sharply decreases. This is a consequence of the formation of crystalline nuclei.

Figure 6 clearly demonstrates the polytetrahedral nature of disordered hard sphere packings. The transition from a 


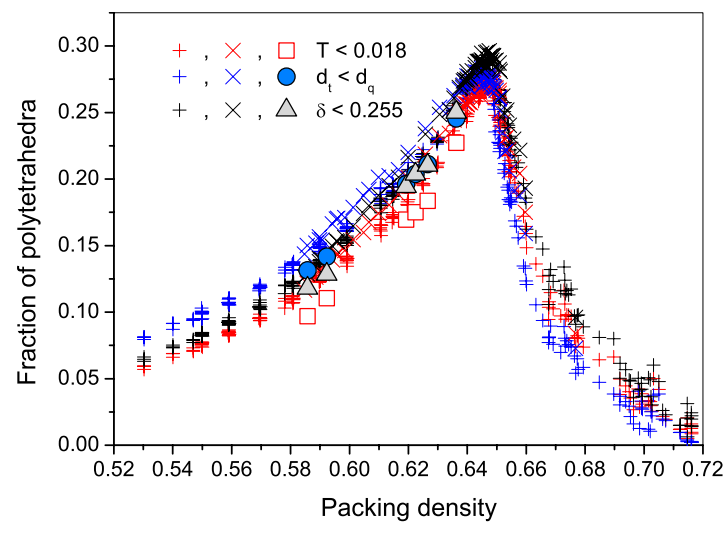

FIG. 6. (Color online) Fraction of quasiperfect tetrahedra which are also part of polytetrahedra aggregates vs packing density. Symbols are as in Fig. 2. The behavior of these curves is quite different from the curves in Fig. 2 revealing a sharp peak at the RCP limiting density.

lower density to higher density packing occurs via increasing the fraction of quasiperfect tetrahedral configurations and their coalescence into polytetrahedral aggregates. At the limiting density $\rho \sim 0.646$ the fraction of quasiperfect tetrahedra reaches its maximum and the polytetrahedral aggregates span their largest extension. Questions then arise: Why this mechanism stops to work for higher densities? What resource of packing becomes exhausted upon approaching the critical density? The answer to these questions might be found in Fig. 7 which demonstrates that the parameter reaching its limit is the number of spheres involved in quasiperfect tetrahedra [9]. For each packing we counted all spheres that are vertexes of at least one quasiperfect tetrahedron. (Recall that each sphere of the packing is a common vertex for several Delaunay simplexes.) As can be seen, the fraction of these spheres grows with increasing density and reaches $100 \%$ at the RCP density. At the limiting density $\rho \sim 0.646$ all spheres in the packing have been involved in the formation of quasiperfect tetrahedra. So a process of densification by means of formation of polytetrahedral nuclei becomes exhausted.

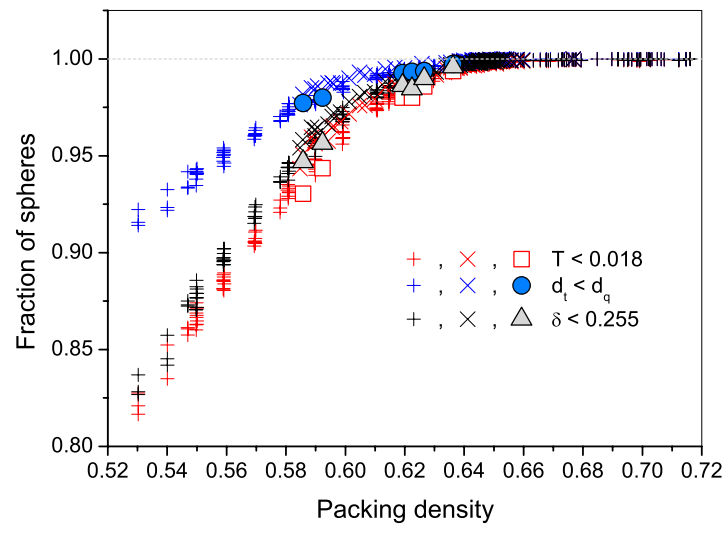

FIG. 7. (Color online) Fraction of spheres in the packing involved in quasiperfect tetrahedra as a function of packing density [9]. Symbols are as in Fig. 2.

\section{ENTROPIC CHARACTERIZATION}

The structural analysis presented in the previous section clearly reveals that a significant reorganization in the packing is occurring at the random-close-packing limit. The open question is now to understand whether such a structural reorganization has the properties of a phase transition. In a classical thermodynamic system, phase transitions occur when at a given temperature, pressure or volume, a new phase becomes more favorable as a result of having a smaller free energy. On the other hand, the sphere packings that we are investigating are dissipative systems that do not conserve energy. They are athermal systems to which the application of thermodynamics concepts is not straightforward. However, in recent years extensions of classical statistical mechanics approaches to these systems have been proposed [42-62]. The main reasoning underlying these approaches relies on the observation of three facts. (1) Mechanically stable packings can be produced with different densities in a wide range between 0.555 to 0.74 by using different preparation methods (or different numerical simulations); (2) for a given preparation procedure, the resulting packing density is highly reproducible within a narrow range of about $0.5 \%$; and (3) the structural properties of the packings at rest are characterized by only a small set of parameters regardless of the preparation procedure $[6,56,57,63,64]$.

The idea is that to this reproducible set of configurations one can apply an extended statistical mechanics reasoning where the role traditionally played by the energy is instead played by the total occupied volume $V[=\pi N /(6 \rho)$, with $N$ the number of spheres] $[21,43,62]$. In this context the canonical ensemble corresponds to a system exploring a set of configurations with average volume $\bar{V}$. This average volume is determined by the exact nature of the preparation procedure. Whereas fluctuations around $\bar{V}$ are associated with the system's entropy.

The applicability of a statistical mechanics approach to our computer simulations and experiments is a "working hypothesis" which is strongly supported by important evidence. In particular, it was shown in Refs. $[21,62,64,65]$ that by using a deductive statistical mechanics approach one can infer precise predictions for the distribution of the volume fluctuations at all levels of investigation from the single grain to the whole system. Within such a framework the probability distribution for a configuration with volume $V$ is $[21,62,64,65]$

$$
p(V)=\frac{\Omega(V)}{\Omega(\chi)} \exp \left(-\frac{V}{\chi}\right),
$$

which recalls the equivalent expression in classical thermodynamics, with $\Omega(V)$ counting all the microscopic configurations occupying a total volume $V, \Omega(\chi)$ the partition function, and $\chi^{-1}$ a Lagrange multiplier which controls the average volume $\bar{V}$. By considering the system as made of a number $k$ of "elementary cells" which can have arbitrary volumes above $v_{\min }$, under the condition that the whole system must occupy a total volume $V$, and by assuming that all the cell properties are either completely determined by the 
volumes $v_{i}$ or they are independent from $v_{i}$, one can write $[62,66] \Omega(V)=\frac{\left(V-k v_{m i n}\right)^{k-1}}{\Lambda^{3 k}(k-1) !}$, with $\Lambda$ a constant analogous to the Debye length. Substituting into Eq. (4) one finds $\chi=(\bar{V}$ $\left.-V_{\min }\right) / k$ and the distribution $p(V)$ is identified as a gamma distribution in the variable $V-V_{\min }$, with a "shape" parameter $k$ and a "scale" parameter $\left(\bar{V}-V_{\min }\right) / k[67]$. Therefore the volume distribution is characterized by the average volume $\bar{V}$ and by the parameter $k$, regardless of the preparation method. The very good agreement between this theoretical prediction and the empirical distributions observed both in experiments and simulations $[21,62]$ is a strong argument in favor of the applicability of this statistical mechanics approach to our packings.

Within the same statistical mechanics approach, we can calculate explicitly the system's entropy [62]:

$$
S=k\left[1+\ln \left(\frac{\bar{V}-V_{\min }}{k \Lambda^{3}}\right)\right],
$$

with $\bar{V}$ the average volume and $V_{\min }=k v_{\min }$ the minimum attainable volume. The entropy Eq. (5) is the Gibbs entropy over all the accessible states associated with a preparation method which yields to an average volume $\bar{V}$. Let us note that, despite the great differences in the physical properties of the systems under investigation (namely, LubachevskyStillinger simulations, Jodrey-Tory models, fluidized bed systems [21], and packings of plastic beads in air [6]), all these systems share a common property: the system's volumes are free to fluctuate and the final packings at rest have average volumes that are determined by the preparation procedure and are independent on the initial configuration.

Let us consider the average entropic contribution from each Voronoï cell. In this case Eq. (5) simplifies because the average volume is directly related to the average density $\bar{V}$ $=\pi /(6 \bar{\rho})$ and the minimum volume is fixed by geometry to the one of a regular dodecahedron $V_{\text {min }}$ $=5^{(5 / 4)} / \sqrt{2(29+13 \sqrt{5})} \simeq 0.694$ [1]. The quantity $k$ can be determined from the standard deviation $\left(\sigma_{v}\right)$ of the Voronoï volume fluctuations by using the relation $[21,62]$

$$
k=\frac{\left(\bar{V}-V_{\min }\right)^{2}}{\sigma_{v}^{2}} .
$$

Typical values of $k$ associated to Voronoï partitions in jammed packings of equal spheres vary in a relatively narrow range between 11 and $14[21,62,66]$. In Ref. [66] it was highlighted that large changes in the value of $k$ are observed near critical densities such as the random-loose and the random-close-packing limits.

\section{ENTROPIC CHANGES ABOVE THE RANDOM- CLOSE-PACKING LIMIT}

Let us now investigate whether there is any sizeable change in the entropy when approaching the random-closepacking limit. Figure 8 shows the behavior of the system's entropy [obtained from Eqs. (5) and (6), by using $\Lambda^{3}=1 / 50$ ]

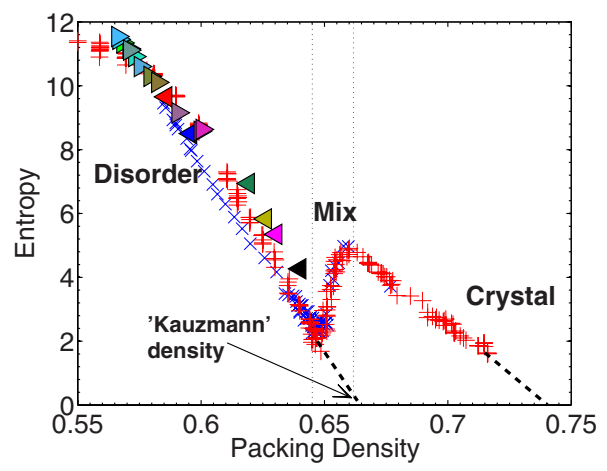

FIG. 8. (Color online) Packing entropy $S$ [Eq. (5)] vs packing density $\rho$ for dry acrylic beads experiments $(\triangleleft)$, glass beads in water prepared by fluidized bed technique $(\triangleright)$, numerical simulations by Jodrey-Tory algorithm $(+)$, and numerical simulations by Lubachevsky-Stillinger algorithm $(\times)$.

as a function of the packing density for all the computer models and the experimental packings $(\mathrm{A}-\mathrm{F})$ considered in the previous sections. Furthermore, Fig. 8 reports data for 12 experiments with glass beads in water [21] also from the AAS database [18]. We observe that all the experiments and simulations fall into a common trend characterized by three distinct regions.

(1) In the density region between the random-loosepacking limit $\rho \sim 0.555$ and the random-close-packing limit $\rho \sim 0.646$ the entropy uniformly decreases.

(2) Just above the random-close-packing limit and precisely between the densities $\rho \simeq 0.646$ and 0.66 the entropy suddenly increases.

(3) After $\rho \simeq 0.66$ the entropy decreases again heading towards zero at the crystalline limit $\rho=0.7405 \ldots$...

The fact that the overall trend of the entropy is decreasing with the density is simple to explain: the more compact the packing becomes, less possibilities are left to the spheres to explore equivalent configurations. In other words the number of degrees of freedom decreases with the density. The remarkable fact is that just above the RCP limit the entropy suddenly increases.

Let us note that the behavior of entropy reported Fig. 8 has strong analogies with what is observed in glass-forming supercooled liquids. If we follow the decreasing trend of the entropy of the disordered packings in the region before the RCP limit $(\rho<0.646)$, we can extrapolate a density where the entropy of the disordered packing will vanish $\left(\rho_{K}\right.$ $\simeq 0.66$, see dashed line in Fig. 8). Such a behavior is very closely related to the Kauzmann paradox in supercooled liquid where, as the temperature decreases, the so-called "configuration entropy" decreases and is extrapolated to zero at a given finite temperature called the Kauzmann temperature [68]. It is usually explained that approaching such a temperature the number of disordered configurations becomes smaller and the relaxation time increases drastically. As a consequence, close to the Kauzmann temperature, it is not longer possible to equilibrate the system on the experimental time scales. Similarly, we may think that as the "Kauzmann density" $\rho_{K}$ is approached, the number of disordered configurations becomes vanishingly small and numerical simula- 
tions are not able to find them any longer. An independent support for such a scenario is given by the analysis of the equation of state in hard sphere simulations by Aste and Coniglio [69] where diverging relaxation times were observed when the packing density approaches the limit $\sim 0.65$. More recently, Kamien and Liu [70] give an estimation of such limiting density at $\sim 0.6465$. Above this density the numerical procedures or the experiments fail in finding disordered packings. Conversely, it becomes more probable to produce phase-separated mixtures of crystalline and disordered phases. As we have seen, this mixing occurs in the range of densities between 0.646 and 0.66 . This is consistent with what was observed from the analysis of the Delaunay simplexes that highlighted the disassemblement of the quasiperfect polytetrahedral aggregates occurring in the same density region between $\rho \simeq 0.646$ and 0.66 (Fig. 2). Above the Kauzmann density $\rho_{K} \simeq 0.66$, only the (poly)crystalline phase is present, and the entropy will vanish at the maximal density $\rho=0.7405 \ldots$.

Let us finally note that there is a change in the behavior of the entropy for both experiments and Jodrey-Tory simulation around $\rho \sim 0.6$, but at this stage we do not have enough data to establish if there is effectively any transition occurring at this density.

\section{CONCLUSIONS}

We have studied structural and entropic properties of equal sphere packings produced by using numerical simulations and experiments. We have shown that at the randomclose-packing limit ( $\rho \simeq 0.646)$ the system undergoes a structural reorganization which is also associated with a sharp increase in the system's entropy.

The structural transition has been studied by using the Delaunay simplex decomposition by measuring, with different criteria, the fraction of tetrahedra with quasiregular shapes. We have shown that, independently of the criterion used to classify shape, the fraction of quasiregular tetrahedra grows with the density reaching a plateau around $30 \%$ at the RCP limit. Interestingly, such a plateau value coincides with the number of tetrahedra present in the Barlow packings. The analysis of the polytetrahedral aggregates (formed by quasiregular tetrahedra which share a common triangular face) reveals that during compaction large clusters of polytetrahedral aggregates (with more than two elements) are formed and they increase in number until the RCP limit where almost all quasiregular tetrahedra are involved in such clusters. The important properties of such aggregates are, on one hand, their rather high local density, and on the other hand their incompatibility with crystalline structures. (In crystals they either contact at edges or are organized in bipyramids.) At the RCP limit all the spheres are involved in quasiregular tetrahedra. This means that the supply for polytetrahedral cluster growth becomes exhausted. Any further increase in density cannot exploit this noncrystalline organization. To reach higher densities the "crystalline" organization has to be exploited. At the RCP limit a sharp transition is observed and the polytetrahedral aggregates are rapidly disassembled. It must be stressed that to observe this sharp structural transition it is important that the basic tetrahedra are quasiregular but not perfect. Indeed, in the configurations that we have considered the gaps between the neighboring spheres may be up to $\sim 25 \%$ of the sphere diameter.

In order to understand the origin of this structural transition in terms of a statistical mechanics perspective, we computed the system's entropy from the measurements of the Vorornoï volume distribution. The entropy of the disordered packings decreases linearly with density, this is analogous with the behavior of the configuration entropy in glassforming supercooled liquids when temperature decreases. The extrapolation of the entropy behavior for the disordered phase indicates that it will vanish at a critical "Kauzmann density" $\rho_{K} \simeq 0.66$. This might indicate that this point identifies a "genuine" phase transition where only one disordered configuration or a nonextensive number of disordered states is left. However, approaching this limit the system fails to find these disordered configurations both in computer simulation and physical experiment. In this condition, mixed disordered-crystalline configurations become more favorable. This mechanism is clearly visible in the region 0.6460.66 , where the polytetrahedral aggregates are disassembled and the entropy has a sharp increase. For densities above this region the packings are in a crystalline phase with an entropy which goes to zero at the densest crystalline packing limit $(0.74 \ldots)$.

\section{ACKNOWLEDGMENTS}

Many thanks to the referees for their careful review and useful suggestions. In particular, we acknowledge the important observation of the second referee which pointed out the analogy between the behavior of the entropy in these packings and the Kauzmann paradox in supercooled liquids. This work was partly supported by RFFI Grants No. 05-03032647 and No. 08-03-00140 (N.M. and A.A.) N.M. acknowledges also travel RFFI Grant No. 06-03-43028 and A.A. acknowledges partial support from YS INTAS No. 0483-3865. T.A. acknowledges the partial support by the ARC discovery project No. DP0450292.
[1] T. Aste and D. Weaire, The Pursuit of Perfect Packing (Institute of Physics, Bristol, 2000).

[2] J. D. Bernal, Nature (London) 183, 141 (1959).

[3] J. D. Bernal and J. Mason, Nature (London) 188, 910 (1960).

[4] G. D. Scott, Nature (London) 194, 956 (1962).
[5] G. Y. Onoda and E. G. Liniger, Phys. Rev. Lett. 64, 2727 (1990).

[6] T. Aste, M. Saadatfar, and T. J. Senden, Phys. Rev. E 71, 061302 (2005).

[7] J. D. Bernal, Proc. R. Soc. London, Ser. A 280, 299 (1964). 
[8] S. Torquato, T. M. Truskett, and P. G. Debenedetti, Phys. Rev. Lett. 84, 2064 (2000).

[9] A. V. Anikeenko and N. N. Medvedev, Phys. Rev. Lett. 98, 235504 (2007).

[10] N. Medvedev and Y. Naberukhin, J. Phys. A 21, L247 (1988).

[11] Y. I. Naberukhin, V. P. Voloshin, and N. N. Medvedev, Mol. Phys. 73, 917 (1991).

[12] W. S. Jodrey and E. M. Tory, Phys. Rev. A 32, 2347 (1985).

[13] A. Bezrukov, M. Bargiel, and D. Stoyan, Part. Part. Syst. Charact. 19, 111 (2002).

[14] K. Lochmann, A. Anikeenko, A. Elsner, N. Medvedev, and D. Stoyan, Eur. Phys. J. B 53, 67 (2006).

[15] M. Skoge, A. Donev, F. H. Stillinger, and S. Torquato, Phys. Rev. E 74, 041127 (2006).

[16] M. D. Rintoul and S. Torquato, Phys. Rev. E 58, 532 (1998).

[17] A. Donev, S. Torquato, and F. H. Stillinger, Phys. Rev. E 71, 011105 (2005).

[18] See, http://wwwrsphysse.anu.edu.au/granularmatter/.

[19] T. Aste, M. Saadatfar, A. Sakellariou, and T. Senden, Physica A 339, 16 (2004).

[20] T. Aste, J. Phys.: Condens. Matter 17, S2361 (2005).

[21] T. Aste, T. D. Matteo, M. Saadatfar, T. Senden, M. Schröter, and H. L. Swinney, Europhys. Lett. 79, 240031 (2007).

[22] M. Schröter, D. I. Goldman, and H. L. Swinney, Phys. Rev. E 71, 030301(R) (2005).

[23] N. N. Medvedev, Voronoi-Delaunay Method for NonCrystalline Structures (SB Russian Academy of Science, Novosibirsk, 2000) (in Russian).

[24] A. Okabe, B. Boots, K. Sugihara, and S. Chiu, Spatial Tessellations-Concepts and Applications of Voronoi Diagrams (Wiley, New York, 2000).

[25] G. F. Voronoï, J. Reine Angew. Math. 134, 198 (1908).

[26] G. F. Voronoï, J. Reine Angew. Math. 136, 67 (1909).

[27] B. N. Delaunay, in Proceedings of the Mathematical Congress Toronto, 1924, (University of Toronto Press, Toronto, 1928), pp. 695-700.

[28] B. N. Delaunay, Izv. Akad. Nauk SSSR, Otd. Mat. Estestv. Nauk 75, 793 (1934).

[29] M. Kimura and F. Yonezawa, J. Non-Cryst. Solids 61-62, 535 (1984).

[30] R. M. Lynden-Bell and P. Debenedetti, J. Phys. Chem. B 109, 6527 (2005).

[31] A. Anikeenko, N. Medvedev, and M. Gavrilova, in Proceedings of the 3rd International Symposium on Voronoi Diagrams in Science and Engineering 2006, edited by B. Werner (IEEE Computer Society, New York, 2006), pp. 148-152.

[32] T. C. Hales, Discrete Comput. Geom. 17, 1 (1997).

[33] A. Anikeenko, M. L. Gavrilova, and N. Medvedev, Lect. Notes Comput. Sci. 3480, 816 (2005).

[34] I. Dryden and K. Mardia, Statistical Shape Analysis (Wiley, New York, 1998).

[35] D. Kendall, D. Barden, T. K. Carne, and H. Le, Shape and Shape Theory (Wiley, Chichester, 1999).

[36] S. Umeyama, IEEE Trans. Pattern Anal. Mach. Intell. 136, 378 (1991).

[37] J. H. Conway and S. Torquato, Proc. Natl. Acad. Sci. U.S.A. 103, 10617, (2006).
[38] J. L. Finney and J. Wallace, J. Non-Cryst. Solids 435, 165 (1981).

[39] F. Frank, Proc. R. Soc. London, Ser. A 215, 43 (1952).

[40] F. Spaepen, Nature (London) 408, 781 (2000).

[41] P. Ganesh and M. Widom, Phys. Rev. B 74, 134205 (2006).

[42] A. Mehta and S. F. Edwards, Physica A 157, 1091 (1989).

[43] S. F. Edwards and D. V. Grinev, Phys. Rev. Lett. 82, 5397 (1999).

[44] A. Barrat, J. Kurchan, V. Loreto, and M. Sellitto, Phys. Rev. Lett. 85, 5034 (2000).

[45] H. A. Makse and J. Kurchan, Nature (London) 415, 614 (2002).

[46] A. Fierro, M. Nicodemi, and A. Coniglio, Europhys. Lett. 59, 642 (2002).

[47] B. Behringer, Nature (London) 415, 594 (2002).

[48] G. D’Anna, P. Mayor, A. Barrat, V. Loreto, and F. Nori, Nature (London) 424, 909 (2003).

[49] Y. Srebro and D. Levine, Phys. Rev. E 68, 061301 (2003).

[50] R. Blumenfeld and S. F. Edwards, Phys. Rev. Lett. 90, 114303 (2003).

[51] S. F. Edwards, J. Brujić, and H. A. Maskse, in Unifying Concepts in Granular Media and Glasses, edited by H. Ed. A. Coniglio, A. Fierro, and M. Nicodemi (Elsevier, Amsterdam, 2004), pp. 9-23.

[52] R. P. Ojha, P. A. Lemieux, P. K. Dixon, A. J. Liu, and D. J. Durian, Nature (London) 427, 521 (2004).

[53] J. H. Snoeijer, T. J. H. Vlugt, M. van Hecke, and W. van Saarloos, Phys. Rev. Lett. 92, 054302 (2004).

[54] P. T. Metzger, Phys. Rev. E 70, 051303 (2004).

[55] P. T. Metzger and C. M. Donahue, Phys. Rev. Lett. 94, 148001 (2005).

[56] P. Richard, M. Nicodemi, R. Delannay, P. Ribiere, and D. Bideau, Nat. Mater. 4, 121 (2005).

[57] M. Pica Ciamarra, A. Coniglio, and M. Nicodemi Phys. Rev. Lett. 97, 158001 (2006).

[58] F. Lechenault, F. da Cruz, O. Dauchot, and E. Bertin, J. Stat. Mech.: Theory Exp. 2006, P07009.

[59] R. Blumenfeld and S. Edwards, Eur. Phys. J. E 19, 23 (2006).

[60] G.-J. Gao, J. Bławzdziewicz, and C. S. O’Hern, Phys. Rev. E 74, 061304 (2006).

[61] M. P. Ciamarra, M. Nicodemi, and A. Coniglio, Phys. Rev. E 75, 021303 (2007).

[62] T. Aste and T. D. Matteo (unpublished) e-print arXiv:0711.3239v1.

[63] T. Aste and T. Senden, Proceedings of Powders \& Grains 2005, edited by H. J. Herrmann and S. McNamara (Taylor and Francis, London, 2005), p. 37.

[64] T. Aste and T. D. Matteo, Eur. Phys. J. E 22, 235 (2007).

[65] T. Aste, Phys. Rev. Lett. 96, 018002 (2006).

[66] T. Aste and T. D. Matteo, Phys. Rev. E 77, 021309 (2008).

[67] R. V. Hogg and A. T. Craig, Introduction to Mathematical Statistics (Macmillan, New York, 1978).

[68] W. Kauzmann, Chem. Rev. (Washington, D.C.) 43, 219 (1948).

[69] T. Aste and A. Coniglio, Europhys. Lett. 67, 165 (2004).

[70] R. D. Kamien and A. J. Liu, Phys. Rev. Lett. 99, 155501 (2007). 